\title{
A dynamic beam splitter using polymer dispersed liquid crystals materials
}

\author{
Marina Riquelme* ${ }^{\mathrm{a} a}$, Manuel Ortuño ${ }^{\mathrm{a}, \mathrm{b}}$, Andrés Márquez ${ }^{\mathrm{a}, \mathrm{b}}$, , Sergi Gallego ${ }^{\mathrm{a}, \mathrm{b}}$, Inmaculada Pascual ${ }^{\mathrm{a}, \mathrm{c}}$, \\ Augusto Beléndez ${ }^{\mathrm{a}, \mathrm{b}}$
}

${ }^{a}$ Science and Technologies Applied Physics Institute, Universidad de Alicante, PO Box 99, 03080

Alicante (Spain); ${ }^{\mathrm{b}}$ Dept, of Physics, Systems engineering \& Signal Theory, Universidad de Alicante, PO Box 99, 03080 Alicante (Spain); ${ }^{\mathrm{c}}$ Dept. of Optics, Pharmacology y Anatomy, Universidad de Alicante, PO Box 99, 03080 Alicante (Spain)

\begin{abstract}
We build a dynamic beam splitter with a holographic optical element (HOE). The laser light goes through the HOE and a fraction of intensity diffracted and transmitted could be tuned by an electric signal. We use holographic polymer dispersed liquid crystals materials. It is made by holographic recording in which the liquid crystal molecules diffuse to dark zones in the diffraction grating and they can be oriented by means of an electric field. The orientation of the liquid crystal produces a refraction index variation which changes the diffraction efficiency and therefore the grating has a dynamic behavior.
\end{abstract}

Keywords: HOE, electro-optical, switching, beam, splitter, holography.

\section{INTRODUCTION}

A holographic optical element (HOE) is an optical element manufactured using holographic techniques capable of controlling both the phase and light intensity. Unlike conventional optical elements which are reflective or refractive, HOEs are diffractive features. Therefore, a simple device can combine several functions as focusing, deflecting and filtering light due to its particular angle and wavelength sensitivity. Thus, HOEs have been successfully incorporated in applications related to the propagation of light in free space or guided wave purposes. In addition to its high spectral selectivity and multifunctionality, they are thin, lightweight, compact and adaptable to the required geometry. This allows experimental setups in confined areas where no conventional optical element could be included.

There are two types of HOEs. On one side are those devices that act as passive and the other operating as active elements. The main difference between them lies in the modulation of the refractive index. While passive elements show a fixed index modulation, active elements change their modulation by applying an external stimulus. Dynamical properties of HOEs allow intensifying either one or the other optical effect without using other device, thus saving time and money. In this sense, to obtain optimum dynamic HOEs optimize the holographic material is a crucial issue.

Photopolymers have been far studied as holographic recording media yielding excellent results. Particularly, HPDLCs are expected to be the most promising materials in tunable holographic devices. HPDLC has unique properties due to the incorporation of liquid crystals as materials susceptible to change its refractive index by applying an external electric field [1-4].

\footnotetext{
* marina.riquelme@ua.es; phone +34 96590 9951; fax +34 965903464
}

Optics and Photonics for Information Processing VI, edited by Abdul A. S. Awwal, Khan M. Iftekharuddin, Proc. of SPIE Vol. 8498, 84980I - (C) 2012 SPIE · CCC code: 0277-786/12/\$18 - doi: 10.1117/12.929538 
It is well known that holograms are recorded diffraction patterns formed by dark and bright areas. When HPDLC prepolymer syrup is illuminated, polymerization process beginning in radiated areas and liquid crystal diffuses to dark zones and monomer to bright regions. Thereby, liquid crystal molecules are confined into small regions or domains producing the Polymeritzation-Induced Phase Separation (PIPS). The size of this small regions or droplets is essential for the proper operate of switching HOEs and is desirable to be between $100 \mathrm{~nm}$ in order to avoid light scattering.

In this work we develop a holographic beam splitter using a HPDLC mixture as recording media. A beam splitter is an optical device that divides incident light in two o more secondary beams. The holographic beam splitter designed here is formed by a diffraction grating operating in Bragg regimen. Therefore, we only have two significant orders propagating throw the sinusoidal grating (one diffracted and the other transmitted), and using an electric field we can control the refraction index modulation, in other words, the intensity of each order.

In this paper, a beam splitter formed by HPDLC materials is studied. We optimize the pre-polymer mixture and then examine electro-optical parameters.

\section{RECORDING MATERIAL}

HPDLC materials are formed by mixtures of monomer and other components. Typically, photoinitiator (also named dye) sensitizes the monomer to be polymerized in a visible spectral range. Few photoinitiators operate between 400 and $700 \mathrm{~nm}$, so research in this area becomes paramount for such devices. In our research, we use Ethyl Eosine photoinitiator,

Another desired feature is that photoinitiator interacts satisfactorily with the coinitiator so as to form free radicals. During this process, coinitiator is the compound that transfers electrons to substance performing as dye forming a NPG radical which will initiate polymerization process. Required coinitiators must be compatible with the other components as well as producing easily a free radical.

As discussed above, it is required that liquid crystal molecules configure suitable droplets. To avoid scattering, drops size must be smaller than the wavelength of illuminating light, maybe over $100 \mathrm{~nm}$. In this sense, the choice of suitable monomer plays an important role since only those monomers with high polymerization rate can configure small enough domains.

An important factor to consider is the monomer functionality. The functionality of the monomer represents the degree of cross-linking of the polymer. Higher functionality corresponds to greater degree of cross-linking in which branched polymer chains are connected to each other. Moreover, lower functionalities are associated with low cross-linking degrees. It has been shown that relatively low functionalities in monomers improve the diffraction efficiency and decrease domain size, although switching performance is worsened. If we study both diffraction and switching features, optimal values for functionality are between 4 and 4.5. Multifunctional acrylic monomers with functionality above 4 seem to fulfill these requirements. Sutherland et al. were the first to use such materials for holographic gratings operating in Bragg regime with satisfactory results [5].

There are several monomers that satisfy our requirements, but this work is concerned in multifunctional acrylic monomer dipentaerythritol penta-/hexa-acrylate (DPHPA) due to it can be easily miscible with the other components of the solution. Even though its functionality is higher than required (in fact, it is between 5 and 6), it exhibits a good optoelectronic performance [6]. 
Furthermore, a second monofunctional monomer type is added so as to conclude polymerization process as well as restricting polymer chains. If we are using acrylic monomers, N-Vinylpyrrolidone (NVP) shows suitable performance concerning optoelectronic issues. NVP enhances diffraction efficiency by decreasing liquid crystal droplet size. White et al. performed an exhaustive study about how NVP influences pre-polymer mixture based on DPHPA for holographic purposes [7].

A very important factor is the liquid crystal. For holographic purposes, ordinary refractive index must match the refractive index of the polymeric material in which it is immersed. Higher diffraction efficiencies require index modulations $(\Delta \mathrm{n})$ as great as possible. In addition, the liquid crystal chosen must be compatible with other components. If they don't, prepolymer suffers phase separation and homogenization becomes more complex. In fact, if the homogenization is unsuccessful, the amount of scattered light increases and optical properties of holographic devices are worsened.

Several chemical additives could be included in prepolymer syrup. A low reactive substance that decreases interactions between liquid crystals and surrounding polymer is required in order to reducing switching voltages. In this sense, a surfactant is a compound that decreases the interfacial tension between both, liquids and solids compounds. Being amphiphilic, that is to say, with two sides, one hydrophilic and the other hydrophobic, this substance allows overcome our limitations.

There are two leading types of liquid crystal used in H-PDLC systems. On the one hand, liquid crystals E7 and BL from Merck, composed by aromatic cyanobiphenyls mixtures, are easily miscible in acrylic monomers as used in this work. On the other, TL liquid crystals from Merck are constituted by combinations of chloro and fluoro substituted messogens show limited solubility in a monomer. Nevertheless, this type decreases the switching voltage in electro optical setup.

In order to improve electro-optical HPDLC beam splitter behavior, we optimize YEt, NPG and NVP taking into account the amounts that improve the diffraction efficiency. Also, the influence of two different liquid crystal types is studied.

An advantage of using the H-PDLC as holographic recording material is the ease of preparation of the prepolymer. First, we introduce the crosslinker NVP in a test tube, since it acts as a solvent for the other substances. Solid elements and liquid crystal are incorporated. Before introducing monomer, surfactant octanoic acid (OA) is added to prevent phase separation in our syrup. All components are mixed with a magnetic stirrer. Finally, polymerizable monomer is added at $40{ }^{\circ} \mathrm{C}$ in order to decrease its viscosity and mixing different compounds. Resulting solution is homogenized in an ultrasonic bath. Prepolymer is sandwiched between two glass slides coated with indium tin oxide (ITO). Microspheres of diameter $13 \mu \mathrm{m}$ are used to ensure conductor faces don't touch each other. A $9.5 \times 10^{4} \mathrm{~Pa}$ pressure is applied to accelerate the formation of the liquid layer between glasses.

We examine degradation processes in prepolymeric material induced by aging and heating.

\section{EXPERIMENTAL SET-UP}

\subsection{Holographic set-up}

To record our beam splitter we use a holographic setup. This setup consists in two beam interference pattern projected on our material. Experimental device is shown in figure 1.

A Nd:YAG laser at a wavelength of $532 \mathrm{~nm}$ was used to store diffraction gratings by means of continuous laser exposure. The primary beam is split into two secondary beams with an intensity ratio of $1: 1$. If each one of these beams goes through a microscope objective, in focal plane the Fourier transform will be obtained with a Gaussian profile. Taking into account that monochromatic plane wave transform is a Dirac's delta, a pinhole is located in order to erase 
undesired frequencies. A lens makes inverse Fourier transform producing finally a monochromatic plan wave. The object and reference beams were interference in the sample at an angle $\theta\left(16^{\circ}\right.$ to the normal) and the spatial frequency obtained is 1036 lines $/ \mathrm{mm}$. The working intensity at $532 \mathrm{~nm}$ is $8.2 \mathrm{~mW} / \mathrm{cm}^{2}$. The diffracted and transmitted intensity are monitored in real time with a He-Ne laser positioned at Bragg's angle $\left(\theta^{\prime}=19.1^{\circ}\right)$ operating to $633 \mathrm{~nm}$, where the material is not sensitive.

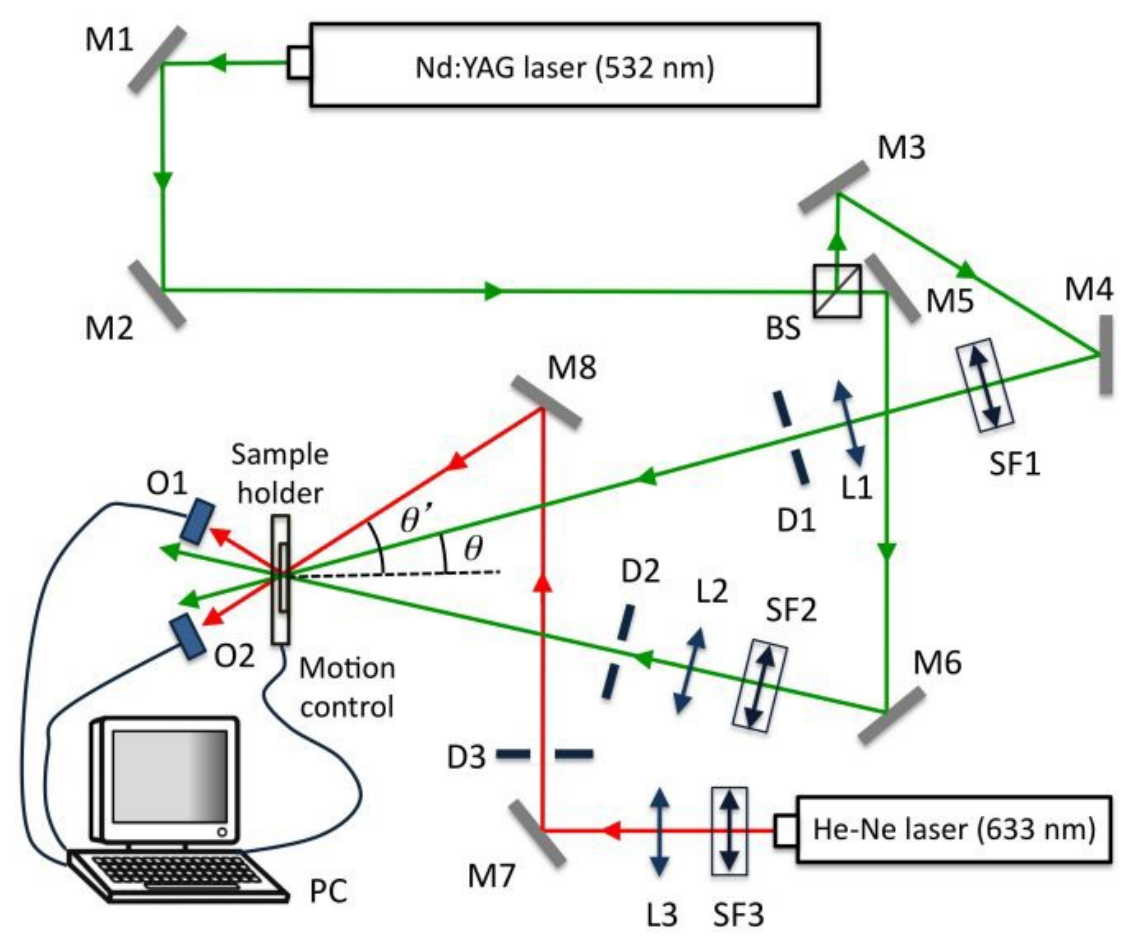

Figure 1. Holographic apparatus: BS beam splitter, Mi mirror, SFi spatial filter, Li lens, Di diaphragm, Oi detectors, PC data recorder

\subsection{Electro-optical measurements}

$\mathrm{AC}$ bipolar square wave voltage is applied to the device after hologram is recorded and the diffracted light is measured. Beam splitter undergoes an electric field in order to study its electro-optical behavior. HPDLC device is driven by $1 \mathrm{KHz}$ AC squared bipolar signal (Tetronik AFG3022B "dual channel arbitrary function generator" $250 \mathrm{MS} / \mathrm{s}, 25 \mathrm{MHz}$ ) connected to a high-voltage amplifier generator (N4L "laboratory power amplifier" LPA 400). Resulting signal is monitorized by an oscilloscope (Tetronik TDS1012B "two channel digital storage oscilloscope" $1 \mathrm{GS} / \mathrm{s}, 100 \mathrm{MHz}$ ).

Holographic beam splitter device is formed by polymer-rich planes parallel to LC-rich planes. Liquid crystal has an electrical charge anisotropy that induces an electrical dipole moment. This charge anisotropy is correlated with a two different refraction index. When electric field is applied, liquid crystal dipole moment beginning to align in its direction with the subsequent LC-rich planes refraction index change. If the refraction index of LC-rich areas match polymer-rich planes index, the recorded grating can be erased. Due to this reason we choose the ordinary LC index near to the surrounding polymeric syrup refractive index [8].

We use a read He-Ne working at $633 \mathrm{~nm}$ laser incident on Bragg angle. Diffracted and transmitted beams are measured to characterize the material behavior when electric field is increased 10 by 10 volts. 


\section{RESULTS AND DISCUSSION}

\subsection{Starting point}

The starting point for our photopolymer materials found in the article ... which suggests the following proportions for components:

Table 1. Starting point table to optimize HPDLC prepolymer compounds.

\begin{tabular}{|c|c|}
\hline Compound & \%wt \\
\hline Monomer & 43.4 \\
\hline LC(E63) & 30 \\
\hline NVP & 17.5 \\
\hline OA & 7 \\
\hline NPG & 1.4 \\
\hline RB & 0.7 \\
\hline
\end{tabular}

Where RB is the Rose Bengal dye. As mentioned above, our blend includes DPHPA as a polymerizable monomer and the liquid crystal BL038 instead E63. Ethyl eosin dye is used unlike that proposed in the previous paper.

Our goal is to improve the holographic behavior of this composition. In this sense, we optimize YEt, NPG and NVP. Also, the influence of liquid crystal type is analyzed. The initial composition with which it comes to optimization photopolymer material is as follows:

Table 2. Starting point table to optimize HPDLC prepolymer compounds

\begin{tabular}{|c|c|}
\hline Compound & \%wt \\
\hline DPHPA & 45,2 \\
\hline LC(BL087) & 27,3 \\
\hline NVP & 17,5 \\
\hline OA & 8,1 \\
\hline NPG & 1,4 \\
\hline Yet & 0,5 \\
\hline
\end{tabular}

There are several criteria to optimize a material; in our case we have chosen the highest value of diffraction efficiency. This parameter is related to the lower values of scattering. We are able to achieve diffraction efficiencies close to $80 \%$ with it.

\subsection{Optimization of the photoinitiator YEt}

The substance used as a photoinitiator absorbs light in a certain range of the visible spectrum in order to initiates polymerization process. Also, its abundance is related with greater light scatter and its shortage with a lower free radical formation. 
Figure 2 shows the diffracted intensity in real time during the recording process of the holographic grating for different YEt concentrations.

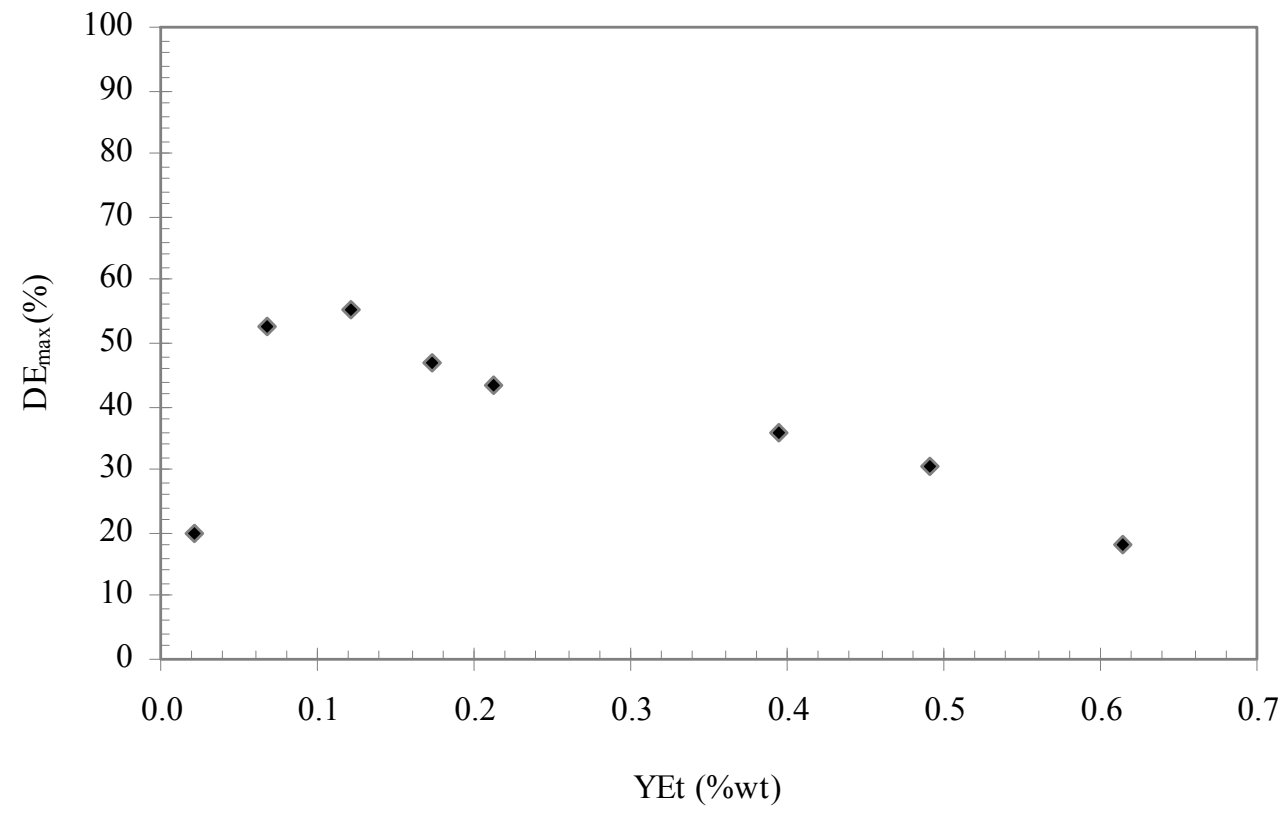

Figure 2. Optimization of photoinitiator Yet.

It is observed that the maximum diffraction efficiency achieved, and therefore, better holographic performance occurs for concentrations around $0.1 \%$ wt. Below this value, the diffraction efficiency decreases because there is not enough dye as to allow formation of initiator molecules of polymer chains. A further increase of the dye concentration decreases proportionately diffraction performance due to a consequent increase in the absorption and scattering of light. As a result, photopolymerization process is hampered and resulting in small diffraction efficiencies.

\subsection{Optimization of coinitiator NPG}

For increase YEt sensitivity, coinitiator may be used. Taking the dye concentration that exhibits the best diffraction efficiency values, we continue with the coinitiator NPG, remaining as fixed parameters both the amount of DPHPA and Ethyl Eosin. 


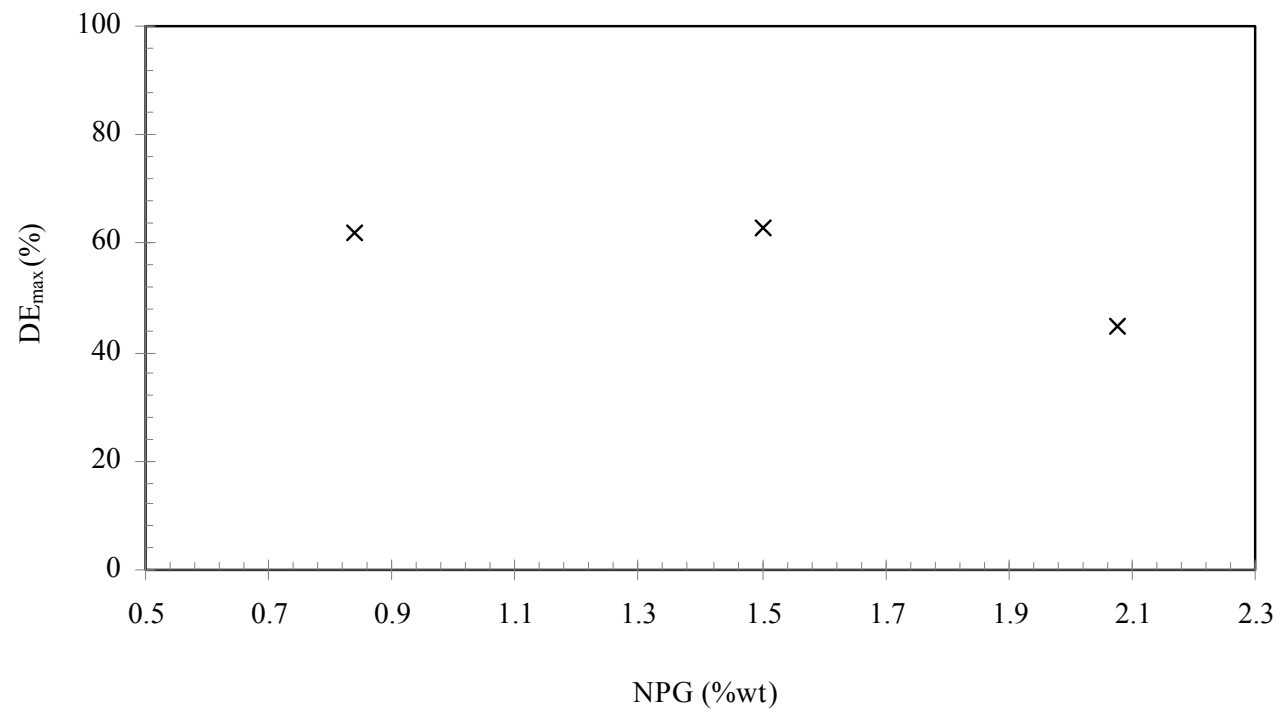

Figure 3. Optimization of coinitiator NPG.

Figure 3 shows the maximum diffraction efficiency as a function of the concentration of coinitiator. It is noted that in the range between $0.8 \%$ and $1.5 \%$ diffraction performance only slightly change, reaching about $62 \%$. A further increase of the concentration involves a decrease in diffraction efficiency.

\subsection{Optimization of NVP}

The next step is to modify the amount of NVP wich works as crosslinker, solubilizer, reducer of oxygen inhibition and is related with the droplet sizes. 


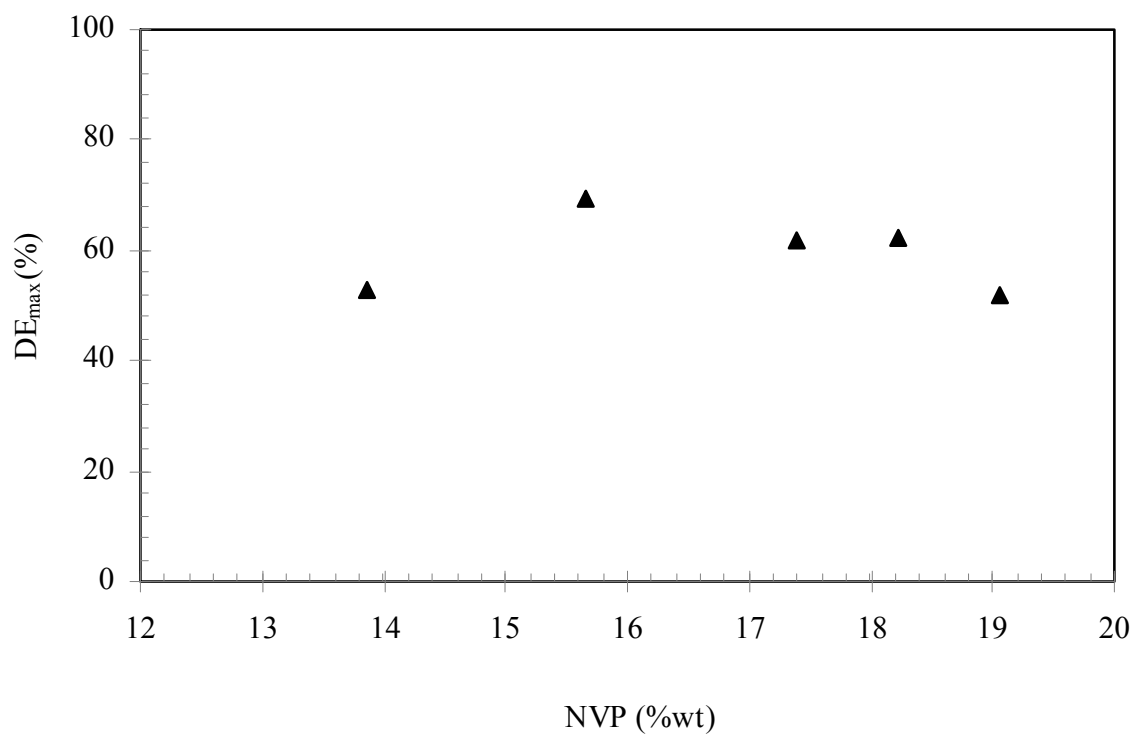

Figure 4. Optimization of NVP cross-linker

From Figure 4, the optimum NVP concentration is into the interval 15-16\%wt. A high or low concentration undergoes a poor diffraction efficiency due to the interaction of the aspects indicated above.

\subsection{Liquid Crystal influence}

During our experiments we have employed two types of liquid crystal, the BL and LT.

An interesting observation in liquid crystal TL213 behavior is that when is included in our solution occurs an instantaneous phase separation in which part of the prepolymer is transformed into solid phase. This fact requires several hours of homogenization by sonication in heated water about $60{ }^{\circ} \mathrm{C}$, so the preparation of the prepolymer solution is more difficult than if we use the type BL038. As for the study of the optical parameters, we consider the modulation index reached by the device is not significantly affected by the type of liquid crystal used as shown in the table below (measurement have all 5\% error).

Table 3. LC type and maximum DE.

\begin{tabular}{|c|c|}
\hline LC type & DEmax(\%) \\
\hline TL213 & 60.8 \\
\hline BL038 & 62.5 \\
\hline
\end{tabular}

\subsection{Prepolymer aging}

The prepolymer solution is stored for six months in a dark place in order to study the prepolymer aging and its influence in the holographic performance. The Figure 5 shows the diffraction efficiency for $200 \mathrm{~mJ} / \mathrm{cm}^{2}$ exposure energy for two types of plates with the same composition but different age. 


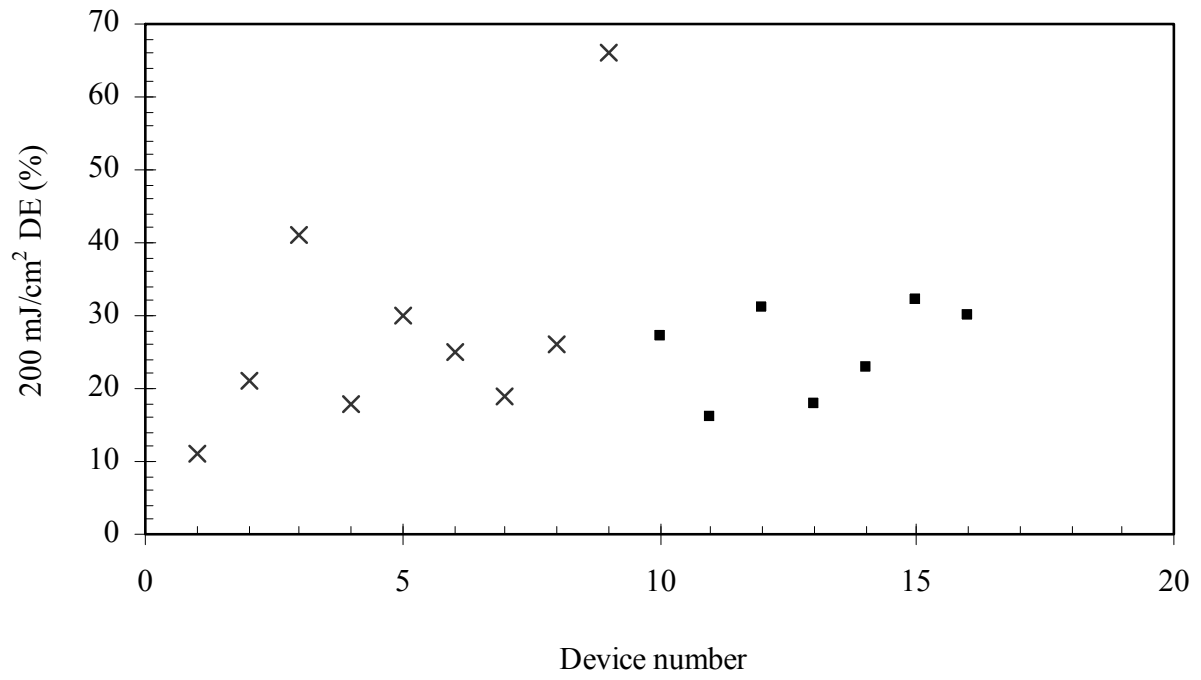

Figure 5. Diffraction efficiency in an exposition of $200 \mathrm{~mJ} / \mathrm{cm} 2$ for a solution stored for six months (crosses) and a freshly prepared (squares).

Both solutions exhibit a similar behavior, oscillating in a range between $10 \%$ and $30 \%$ in terms of diffraction efficiency. The values of diffraction efficiency do not change significantly after a prolonged storage of photopolymer mixture in varying ambient temperature conditions $(10 \%-30 \%)$ and in the dark.

Another way of degradation of the photopolymer mixture is the thermal effect. Figure 6 shows the results after increasing the temperature of the solution from $20{ }^{\circ} \mathrm{C}$ to $53{ }^{\circ} \mathrm{C}$.

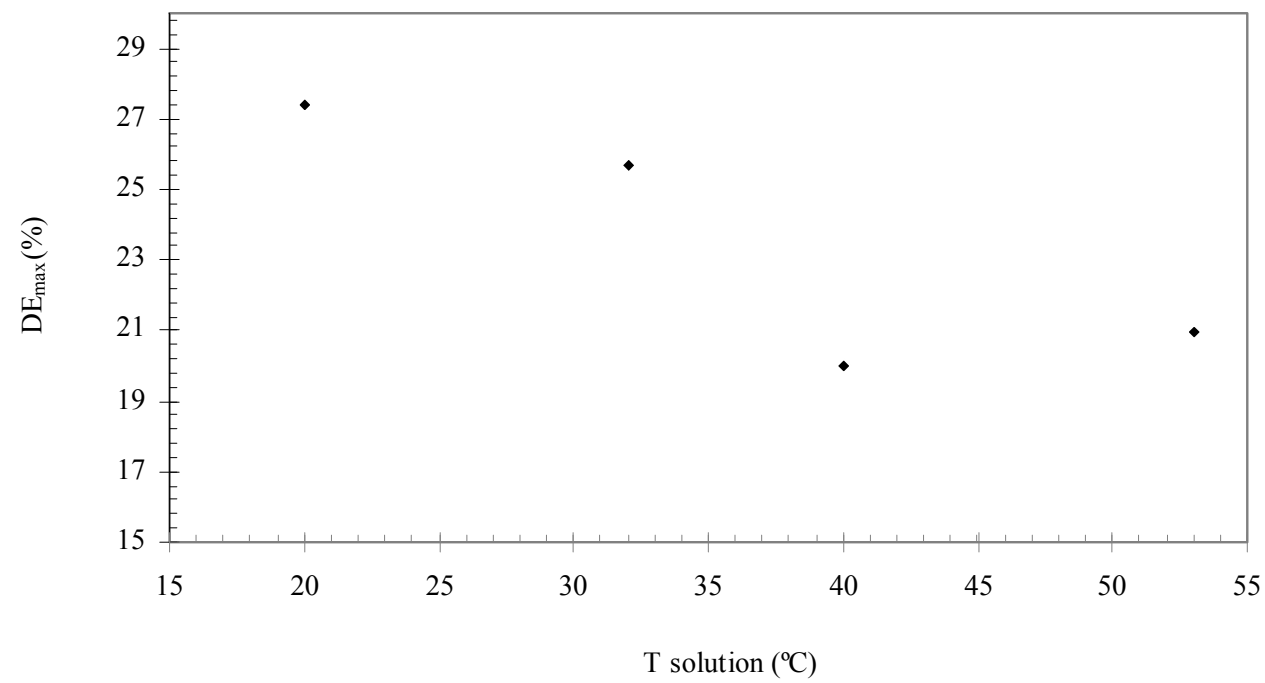

Figure 6. Degradation of the photopolymer mixture by thermal effect.

Proc. of SPIE Vol. 8498 84980I-9 
As can be seen, the maximum diffraction efficiency recorded decreases from $27 \%$ to $20 \%$ when the temperature increases from $20^{\circ} \mathrm{C}$ to $53{ }^{\circ} \mathrm{C}$. The observed effect is attributable to a partial polymerization of the mixture by heating, resulting in a decrease of the diffracted light during holographic exposure. A high temperature produce chemical reactions between the active components of the mixture.

\subsection{Electro-optical performance}

The diffraction efficiency decreases by about $70 \%$ of its initial value when an electric field is applied. This is caused by the reordering of the liquid crystal molecules present in the unexposed areas of the diffraction grating. Liquid crystal molecules have net dipole moment and applying the electric field tends to align its main axis in the direction of the field. This involves changes in the refractive index modulation of the plate (Figure 7).

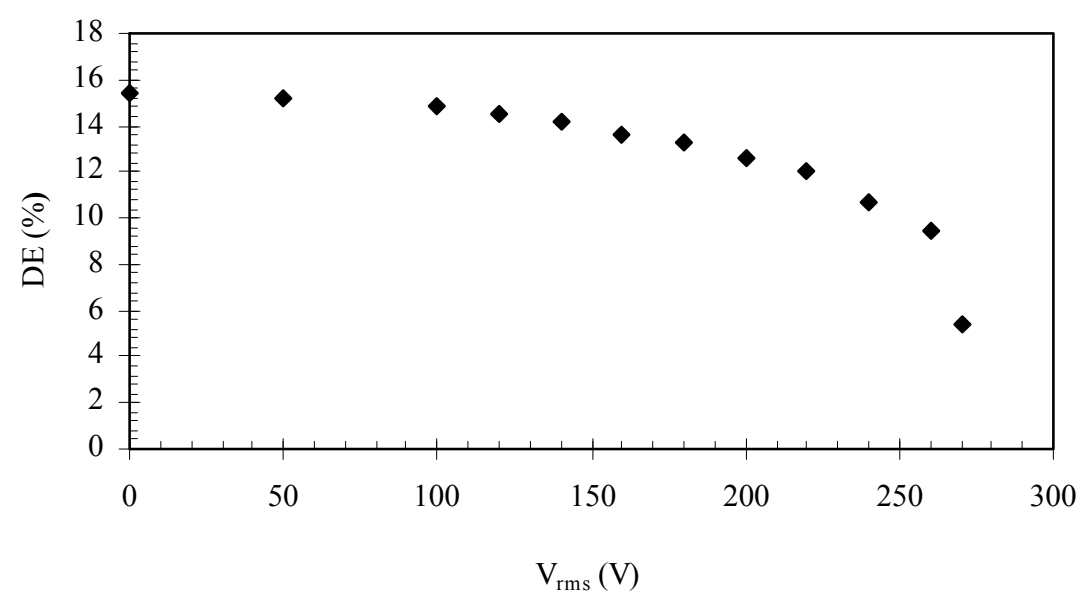

Figure 7. Variation in diffraction efficiency as a function of applied electric field

Before reaching 100 Vrms DE changes very weak. From this value a gradual decrease, with the steepest decline from the $250 \mathrm{Vrms} . \mathrm{DE}=0 \%$ is not reached because refractive indices of the exposed and unexposed areas are not matching. Furthermore, for high values of electric fields, the plate, which initially acts as a condenser, begins to conduct, raising its temperature by ohmic effect, resulting in different degradative effects.

\subsection{LC influence in electro-optical behavior}

The following measurements are performed for linearly polarized incident light vertically (Figure 8). The device prepared from a solution including the liquid crystal TL213 varies very little its performance in a diffraction range between $0 \mathrm{~V}$ and $90 \mathrm{~V}$. From this last value, the diffraction efficiency decreases by $17 \%$ to $110 \mathrm{~V}$, where stops working. In the same range of applied potential, the holographic grating containing the BL038 liquid crystal reduces its initial diffraction efficiency $90 \%$, reaching transparency $(\mathrm{RD}=0 \%)$ for $110 \mathrm{~V}$. 


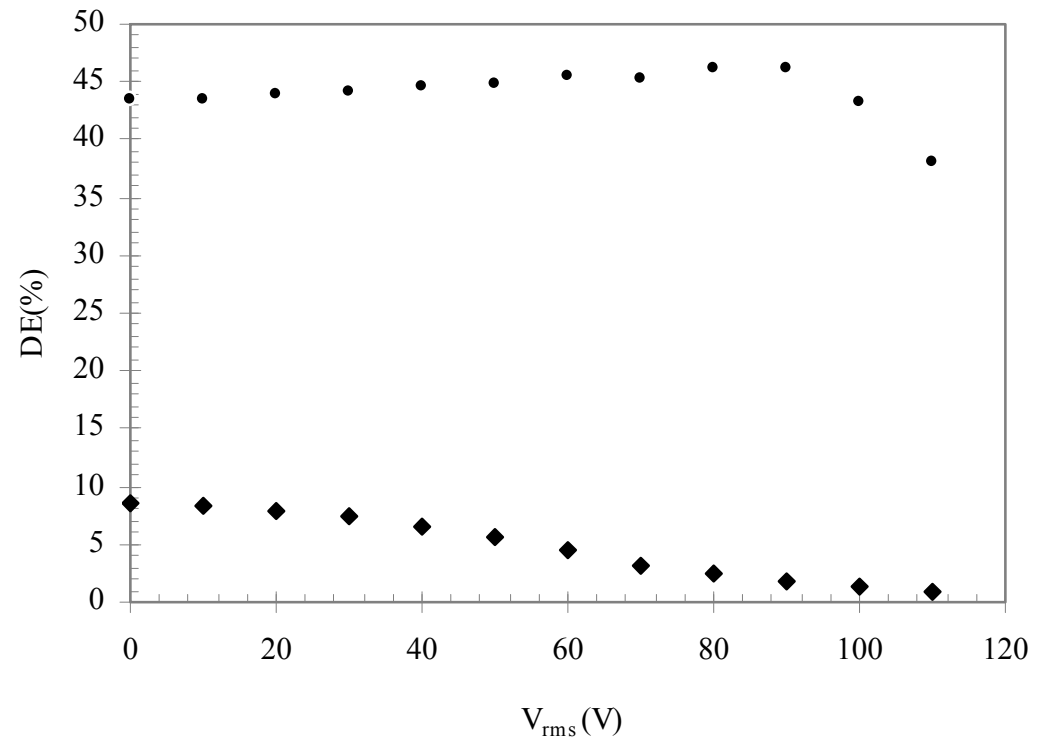

Figure 8.Variation in diffraction efficiency for two different liquid crystal types: TL213 (circles) and BL038 (rhombs).

\section{REFERENCES}

[1] Mehta, P.C. and Rampal, V.V., [Lasers and Holography], World Scientific Publishing Co. Pte. Ltd., Farrer Road, Singapore, 517-519 (1993).

[2] Bunning, T. J., Natarajan, L.V., Tondiglia, V.P., and Sutherland, "Holographic Polymer-Dispersed Liquid Crystals (HPDLCs)," R.L., Annu. Rev. Mater. Sci. 30, 83-115 (2000).

[3] Liu, Y.J., and Sun, W., "Holographic Polymer-Dispersed Liquid Crystals: Materials, Formation and Applications," Advances in OptoElectronics, 2008, 1-52 (2008).

[4] Liu, Y.J., Zhang, B., Jia, Y., and Xu, K., "Improvement of the diffraction properties in holographic polymer dispersed liquid crystal Bragg gratings," Optics Comunications 218, 27-32 (2003).

[5] Pogue, R.T., Natajaran L.V., Siwecki, S.A., Tondiglia, V.P., Sutherland, R.L., and Bunning, T. J.," Monomer functionality effects in the anisotropic phase separation of liquid crystals", Polymer, 41(2) 733-741 (2000).

[6] Sutherland, R. L., Natarajan, L. V., Tondiglia, V. P., Bunning, T. J., "Bragg Gratings in an Acrylate Polymer Consisting of Periodic Polymer-Dispersed Liquid-Crystal Planes," Chem. Mater. 5, 1533-1538 (1993).

[7] White, T.J, Liechty, W.B., Natarajan, L. V., Tondiglia, V. P., Bunning, T. J., and C.A. Guymon, "The Influence of N-Vinyl-2-Pyrrolidone in Polymerization of Holographic Polymer Dispersed Liquid Crystals (HPDLCs)," Polymer 47 (7), 2289-2298 (2006).

[8] Natarajan, L. V., Sutherland, R. L., Tondiglia, V. P., Bunning, T. J., "Electro-optical switching characteristics of volume holograms in polymer dispersed liquid crystals," J. Nonlinear Opt. Phys. Mater. 5, 89-98 (1996). 